\title{
Diffusing diffusivity: a new derivation and comparison with simulations $^{\dagger}$
}

\author{
ROHIT JAIN ${ }^{\mathrm{a}}$ (D) and K L SEBASTIAN ${ }^{\mathrm{a}, \mathrm{b}, *}$ \\ ${ }^{a}$ Department of Inorganic and Physical Chemistry, Indian Institute of Science, Bangalore, Karnataka 560012 , \\ India \\ b Indian Institute of Technology Palakkad, Ahalia Integrated Campus, Kozhippara, Palakkad, Kerala 678 557, \\ India \\ E-mail: klsiisc@gmail.com
}

MS received 29 January 2017; revised 2 May 2017; accepted 2 May 2017

\begin{abstract}
Many experiments are now available where it has been shown that the probability distribution function (pdf) for the position of a Brownian particle diffusing in a heterogeneous medium is not Gaussian. However, in spite of this non-Gaussianity, the mean square displacement (MSD) still remains Fickian, i.e., $\left\langle x^{2}\right\rangle \propto T$. One possible explanation of this non-Gaussian yet Brownian behavior is that the diffusivity of the particle itself is "diffusing". Chubynsky and Slater (Phys. Rev. Lett. 113098302 2014) proposed a model of "diffusing diffusivity" which they were able to solve analytically at small time scales, but simulations were performed for intermediate to large time scales. We present here a class of diffusing diffusivity models and show that the problem of calculating pdf for the position of diffusing particle is equivalent to calculating the survival probability of a particle undergoing Brownian motion in the presence of a sink. We give exact analytical results for all time scales and show that the pdf is non-Gaussian at short times which crosses over to a Gaussian at long times. The MSD is also shown to vary linearly with time at all times. We find that our results reproduce the numerical results of Chubynsky and Slater quite well.
\end{abstract}

Keywords. Diffusing diffusivity; anomalous yet Brownian; non-Gaussian parameter.

\section{Introduction}

The theory of Brownian motion was formulated by Einstein in $1905^{1}$ while studying the irregular movement of microscopic particles suspended in a solvent (see the reviews by Chandrasekhar ${ }^{2}$ as well as by Frey and Kroy. ${ }^{3}$ ) In formulating the theory, it was assumed that the time scale of motion of the particle is much larger than that of the solvent molecules, thereby causing a complete separation of time scales. In general, there will be hundreds of collisions between the particle and the solvent molecules with in the time period in which the particle covers a distance comparable to its size. This separation of time scales leads to two major results: (i) the mean square displacement (MSD) of the particle increases linearly with time, i.e.,

$$
\left\langle x^{2}\right\rangle \propto t
$$

which has been referred to as Fickian diffusion and (ii) the probability distribution function (pdf) of displacements of particle is Gaussian, owing it to the central

\footnotetext{
*For correspondence

† Dedicated to the memory of the late Prof. Charusita Chakravarty.
}

limit theorem. In one dimension this means that the probability of finding a particle, which was at the origin initially, at the position $x$ at a time $t$ is given by

$$
P(x, t)=\frac{1}{\sqrt{4 \pi D t}} e^{-x^{2} / 4 D t} .
$$

Recently it has been realized that there are phenomena, especially for diffusion processes in crowded or active media, where the relation of Eq.(1) is not valid at all times. ${ }^{4-6}$ For many processes, $\left\langle x^{2}\right\rangle \propto t^{\alpha}$ with $\alpha \neq 1$ and the diffusion is termed as anomalous. The motion is called superdiffusive or hyperdiffusive if $\alpha>1$. The superdiffusion or the enhanced diffusion is usually associated with an activated process where diffusion is assisted by an external force. It was first observed by Bouchaud et al., ${ }^{7}$ for a tracer particle in polymer-like, breakable micelles. More details can be found in the review article by Bouchaud and Georges. ${ }^{8}$ Due to the dynamic nature of micellar solutions, the tracer rides micelles of different sizes, thus making its apparent diffusion coefficient change with time. They observed Lévy flight like motion of the tracer. Superdiffusive motion was further seen in the 
simulations of a tracer particle diffusing in crowded environment of randomly moving barriers, ${ }^{9}$ for diffusion in a $2 \mathrm{D}$ complex plasma ${ }^{10}$ and for activated motion in live cells. ${ }^{11}$ Recently, we ${ }^{12}$ gave a model of "diffusing diffusivity" where diffusion coefficient is modeled as a Lévy flight process which eventually leads to superdiffusion for the particle's position. On the other hand if $\alpha<1$, then the motion is called subdiffusive and is usually observed for diffusion of a particle inside crowded environments. While superdiffusion is rare in physical systems, subdiffusion is more frequent and hence a topic of current reasearch interest. It has now been reported in many experiments viz., tracer diffusion in cellular environments, ${ }^{13-16}$ diffusion of fluorescent beads in complex fluids, ${ }^{17}$ receptor diffusion on a cell membrane, ${ }^{18}$ etc and also in several theoretical studies. ${ }^{4,19-21}$ The two most commonly used models to explain subdiffusive motion are that of continuous time random walk (CTRW) ${ }^{22,23}$ and fractional Brownian motion (fBM). ${ }^{24}$ CTRW model is based on a broad distribution of dwell times and hence can be easily distinguished from fBM by testing non-ergodicity. ${ }^{25,26}$ More details on the topic of anomalous transport in crowded media can be found in the nice review article by Höfling and Franosh. ${ }^{5}$

Apart from the non-linear scaling of MSD with time in anomalous diffusion, the pdf is not usually Gaussian. This is so well accepted that it is often assumed that if the pdf is found to be non-Gaussian then the MSD should also be non-linear. Also, it is assumed that if the diffusion of the particle is Fickian, i.e., the MSD is proportional to time then the underlying pdf should be Gaussian. However, with the advances in imaging techniques, it has now become possible to follow the trajectory of a single particle and hence measure the distribution of displacements experimentally. In a recent series of experiments from the group of Granick, ${ }^{27-29}$ it was observed that the MSD is linear over the entire experimental time range, yet the pdf of displacements is non-Gaussian. Banks et al., ${ }^{30}$ in a very recent study have used variable length-scale fluoroscence correlation spectroscopy (VLFCS) to study the diffusion of protein molecules in crowded fluids and have shown that the diffusion is Brownian yet anomalous. Sung et al. ${ }^{31}$ performed simulations to study the rotational diffusion of dumbbells in 2D porous media of stationary hard disks. They showed that for a low matrix density, the system is ergodic due the presence of a percolating network and that the pdf of angular displacements is Gaussian. On the other hand for a high matrix density, the percolating network disappears leaving the system non-ergodic and the ensemble averaged pdf becomes non-Gaussian. This kind of Brownian, yet anomalous diffusion of particles in complex fluids now seems to be a generic phenomenon and a large body of evidence from various fields including many physicochemical and socio-economic processes is available. These processes include hard sphere diffusion in colloidal suspensions, ${ }^{29,32,33}$ liposome diffusion in entangled actin, ${ }^{28}$ lateral diffusion of receptor molecules in cell membranes, ${ }^{18}$ tracer diffusion in crowded media of either larger spheres ${ }^{33,34}$ or polymers, ${ }^{35,36}$ diffusive motion in supercooled liquids ${ }^{37}$ and in systems close to glass and jamming transitions, ${ }^{38,39}$ orientational diffusion of dye molecules in polymer thin films, ${ }^{40,41}$ simulations of $2 \mathrm{D}$ colloids ${ }^{42}$ and hard sphere fluids ${ }^{43}$, price dynamics, ${ }^{44}$ and financial returns. ${ }^{45}$ The salient features of this kind of diffusion processes have been summarised in a recent review by Metzler ${ }^{46}$ and also in the paper by Cherstvy and Metzler. ${ }^{47}$

In many heterogeneous systems, the environment changes slowly either on its own or because the diffusing particle has moved to a different place. For either case, the diffusivity changes as the particle feels a different "local" environment. In the "diffusing diffusivity" model of Chubynsky and Slater, ${ }^{49}$ the authors assume that the environment rearranges over a time scale larger than that of the diffusion process, and therefore it is possible to define a diffusion coefficient unambiguously and that even the variations in diffusion coefficient as the particle moves from one spatial region to another, can be captured by a stochastic $D(t)$. In their study, Chubynsky and Slater showed analytically that at short times, the pdf of displacements is exponential if one assumes an exponential distribution for $D$. However, for long times they had to perform simulations and the result was a cross over to Gaussian. In a recent work, ${ }^{50}$ we gave a general class of "diffusing diffusivity" models which can be solved analytically. With our model, we were able to find complete analytical solutions which could explain all the limits observed in experiments/simulations. Later, we applied this model to calculate the survival probability for a particle diffusing in a crowded, rearranging and bounded domain. ${ }^{51}$ In a very recent study, the same results were obtained by Chechkin et al., ${ }^{48}$ via a different approach known as the subordination method in probability theory.

In this paper, we present our model of "diffusing diffusivity" in which the diffusivity of the particle changes as a random function of time. The details of the model are to be found in our earlier work. ${ }^{50}$ In this paper, we used a path integral approach to show that the problem of calculating the pdf for particle displacement can be reduced to the problem of calculating the survival probability of a fictitious particle (which actually is the diffusion coefficient!) undergoing overdamped Brownian motion in 
the presence of a sink. We also give an exact expression for the non-Gaussianity parameter which is often used as a measure to quantify the extent of deviation from Gaussian distribution. This parameter is zero if the distribution is Gaussian but is non-zero if there is any effect of spread in the diffusivity on the pdf of particle displacement. In this paper, we give a simple, elegant method to show that the problem of calculating the pdf can be reduced to that of random walk with absorption.

The paper is organized in the following manner. In Section 2, we set up the problem by arguing that the diffusivity becomes a stochastic function of time for diffusion processes in complex fluids. In Section 3, we present our model and give an outline to arrive at the solution. We also discuss the results of the model here. In Section 4, we make an explicit comparison of our model with that of Chubynsky and Slater. ${ }^{49}$ We find here that the agreement of our analytical results with their simulation data is quite astonishing despite the differences between the two models. Finally, in Section 5, we present a summary of the paper.

\section{Brownian motion in a crowded, rearranging medium}

In the overdamped limit, the position $x(t)$ of a Brownian particle undergoing diffusion is governed by the stochastic differential equation or the Langevin equation as

$$
\dot{x}(t)=\eta(t),
$$

$\eta(t)$ is the random noise arising from the collisions of solvent molecules with the diffusing particle. In the conventional theory of Brownian motion, it is assumed to have a very short correlation time, $t_{c}$ in comparison with the time scale $t_{B}$ at which the motion of the Brownian particle takes place. i.e., $t_{B} \gg t_{c}$. In this limit, it is appropriate to assume that $\eta(t)$ is a Gaussian white noise having the correlation function

$$
\left\langle\eta(t) \eta\left(t^{\prime}\right)\right\rangle=2 D \delta\left(t-t^{\prime}\right) .
$$

Here $D$ is the diffusion coefficient and the symbol $\langle\cdots\rangle$ stands for averaging over the noise $\eta(t)$. The pdf for finding the particle at $x$ at the time $t, P(x, t)$ obeys the diffusion equation

$$
\frac{\partial}{\partial t} P(x, t)=D \frac{\partial^{2}}{\partial x^{2}} P(x, t) .
$$

We use the bra-ket formalism of quantum mechanics, as that is very convenient for the following analysis. Thus we write the above equation as

$$
\frac{\partial}{\partial t} P(x, t)=-D \hat{p}^{2} P(x, t)
$$

where $\hat{p}=-i \frac{\partial}{\partial x}$ is the momentum operator. As is usual in quantum mechanics, we will also use the eigenkets of momentum and position operators, defined by

$$
\hat{p}|p\rangle=p|p\rangle \text { and } \hat{x}|x\rangle=x|x\rangle
$$

which satisfy $\langle x \mid p\rangle=\frac{1}{\sqrt{2 \pi}} e^{i p x}$.

We now consider the Brownian motion of the particle in an environment which undergoes structural rearrangements. Consider for such a system that the environment rearrangement happens on a time scale $\tau_{R}$ and the shortest interval of observations is $t_{o b s}$. We consider only the case $t_{o b s} \gg \tau_{R}$ so that one can define an instantaneous diffusion coefficient $D$. Thus the rearrangement of the environment can be modelled by taking diffusion coefficient to be a random function of time. In such a case, we have to solve the diffusion equation corresponding to a time-dependent diffusion coefficient

$$
\frac{\partial}{\partial t}|P(t)\rangle=-D(t) \hat{p}^{2}|P(t)\rangle
$$

where $|P(t)\rangle$ is the ket defined by

$$
\langle x \mid P(t)\rangle=P(x, t)
$$

This equation can be formally solved to get $|P(T)\rangle$ at the final time $t=T$ as

$$
|P(T)\rangle=e^{-\int_{0}^{T} d t D(t) \hat{p}^{2}}|P(0)\rangle .
$$

Hence,

$$
P(x, T)=\langle x \mid P(T)\rangle=\left\langle x\left|e^{-\int_{0}^{T} d t D(t) \hat{p}^{2}}\right| P(0)\right\rangle
$$

Introducing the resolution of identity

$$
I=\int_{-\infty}^{\infty} d p|p\rangle\langle p|,
$$

we can write the above equation as

$$
P(x, T)=\langle x \mid P(T)\rangle=\int_{-\infty}^{\infty} d p\langle x \mid p\rangle e^{-p^{2} \int_{0}^{T} d t D(t)}\langle p \mid P(0)\rangle .
$$

Assuming the particle to be at $x=0$ initially (i.e., $P(x, 0)=$ $\delta(x)$, we get

$$
P(x, T)=\int_{-\infty}^{\infty} \frac{d p}{2 \pi} e^{i p x} e^{-p^{2} \int_{0}^{T} d t D(t)} .
$$

As $D(t)$ changes randomly, we average over all realizations of it to get

$$
P(x, T)=\int_{-\infty}^{\infty} \frac{d p}{2 \pi} e^{i p x}\left\langle e^{-p^{2} \int_{0}^{T} d t D(t)}\right\rangle_{\mathcal{D}}
$$

where $<\ldots>_{\mathcal{D}}$ is used to denote averaging over all realisations of $D(t)$. Hence calculation of $P(x, T)$ is reduced to the calculation of the functional

$$
\mathcal{F}(p, T)=\left\langle e^{-p^{2} \int_{0}^{T} d t D(t)}\right\rangle_{\mathcal{D}} .
$$

As $D(t)$ evolves in a random fashion, in the following we take its distribution function $\pi(D, t)$ to obey a Fokker-Planck equation. Hence, the calculation of the functional $\mathcal{F}(p, T)$ can be performed easily, by introducing the sink function $p^{2} D$ into the Fokker-Planck equation (see below). In the following section, we present a model in which $\mathcal{F}(p, T)$ can be calculated analytically. With this model we show that though the 
mean square displacement is linear in time at all times, the distribution, $P(x, T)$ is non-Gaussian at short times which crosses over to being Gaussian in the limit of long time.

\section{Fokker-Planck equation for the evolution of $D(t)$}

We now model $D(t)$ as

$$
D(t)=\xi^{2}(t)
$$

where $\boldsymbol{\xi}=\left\{\xi_{1}(t), \xi_{2}(t), \ldots \xi_{n}(t)\right\}$ is an $n$-dimensional random vector. Since $D(t)$ is obviously constrained to be positive, $\xi^{2}(t)$ being the norm of a vector satisfies this condition. Further we take $\xi(t)$ to be the position vector of an $n$-dimensional harmonic oscillator that is undergoing overdamped Brownian motion. The frequency associated with each direction is taken to be $\omega$ and the "diffusion coefficient" is assumed $F$, which is also same for every direction. The Langevin equation that $\xi_{j}(t)$ obeys is taken to be

$$
\dot{\xi}_{j}(t)+\omega \xi_{j}(t)=\zeta_{j}(t),
$$

where $\zeta_{j}(t)$ is the Gaussian white noise with the correlation function

$$
\left\langle\zeta_{i}(t) \zeta_{j}\left(t^{\prime}\right)\right\rangle=2 F \delta_{i j} \delta\left(t-t^{\prime}\right)
$$

The propagator for the time evolution of $\xi_{j}(t)$ using the method of phase-space path integral ${ }^{52,53}$ is given as

$$
\begin{aligned}
G\left(\xi_{j f}, T \mid \xi_{j 0}, 0\right)= & \int_{\xi_{j}(0)=\xi_{j 0}}^{\xi_{j}(T)=\xi_{j f}} \mathcal{D} \xi_{j}(t) \int \mathcal{D} \pi_{j}(t) \\
& \times e^{-F \int_{0}^{T} d t \pi_{j}^{2}(t)} e^{-i \int_{0}^{T} d t \pi_{j}(t)\left(\dot{\xi}_{j}(t)+\omega \xi_{j}(t)\right)},
\end{aligned}
$$

where $\pi_{j}(t)$ for $j=1,2, \ldots, n$ is the conjugate momentum associated with $\xi_{j}(t)$. The propagator $G\left(\xi_{j f}\right.$, $\left.T \mid \xi_{j 0}, 0\right)$ gives the probability of oscillator to be present at $\xi_{j f}$ at the time $T$ given that at $t=0$ it was at $\xi_{j 0}$. This can be evluated exactly to get

$$
\begin{aligned}
G\left(\xi_{j f}, T \mid \xi_{j 0}, 0\right)= & \sqrt{\frac{\omega}{2 \pi F\left(1-e^{-2 \omega T}\right)}} \\
& \times e^{-\frac{\omega\left(\xi_{j f}-\xi_{j 0} e^{-\omega T}\right)^{2}}{2 F\left(1-e^{-2 \omega T}\right)}} .
\end{aligned}
$$

The details of the method can be found elsewhere (for instance see the paper by Janakiraman and Sebastian ${ }^{52}$ and also the book by Kamenev. ${ }^{53}$ ) Further the distribution for the initial position, $\xi_{j 0}$ can be assumed to be the equilibrium one. As we are not interested in the final value of $D$ at the time $T$, we integrate over all possible final values of $\xi_{j f}$. With these, the average $\langle\ldots\rangle$ in Eq.(13) becomes

$$
\begin{aligned}
\mathcal{F}(p, T)= & \left\langle e^{-p^{2} \int_{0}^{T} d t D(t)}\right\rangle_{\mathcal{D}} \\
= & \prod_{j=1}^{n} \int_{-\infty}^{\infty} d \xi_{j f} \int_{-\infty}^{\infty} d \xi_{j 0} \\
& \times \pi_{j, e q}\left(\xi_{j 0}\right) \int_{\xi_{j}(0)=\xi_{j 0}}^{\xi_{j}(T)=\xi_{j f}} \mathcal{D} \xi_{j}(t) e^{-p^{2} \int_{0}^{T} d t \xi_{j}^{2}(t)} \\
& \times \int \mathcal{D} \pi_{j}(t) e^{-F \int_{0}^{T} d t \pi_{j}^{2}(t)} \\
& \times e^{-i \int_{0}^{T} d t \pi_{j}(t)\left(\xi_{j}(t)+\omega \xi_{j}(t)\right)}
\end{aligned}
$$

The pdf can now be written as

$$
\begin{aligned}
P(x, T)= & \int_{-\infty}^{\infty} \frac{d p}{2 \pi} e^{-i p x} \prod_{j=1}^{n} \int_{-\infty}^{\infty} d \xi_{j f} \int_{-\infty}^{\infty} d \xi_{j 0} \\
& \times \pi_{j, e q}\left(\xi_{j 0}\right) P_{j}^{\operatorname{sink}}\left(\xi_{j f}, T \mid \xi_{j 0}, 0 ; p\right),
\end{aligned}
$$

where $P_{j}^{s i n k}\left(\xi_{j f}, T \mid \xi_{j 0}, 0 ; p\right)$ is given by

$$
\begin{aligned}
P_{j}^{\text {sink }} & \left(\xi_{j f}, T \mid \xi_{j 0}, 0 ; p\right) \\
= & \int_{\xi_{j}(0)=\xi_{j 0}}^{\xi_{j}(T)=\xi_{j f}} \mathcal{D} \xi_{j}(t) \int \mathcal{D} \pi_{j}(t) \\
& \times e^{-\int_{0}^{T} d t\left\{p^{2} \xi_{j}^{2}(t)+F \pi_{j}^{2}(t)+i \pi_{j}(t)\left(\dot{\xi}_{j}(t)+\omega \xi_{j}(t)\right)\right\}} .
\end{aligned}
$$

At this point we introduce the dimensionless quantities defined by, $\tilde{t}=\omega t, \tilde{D}=\frac{\omega}{F} D, \tilde{x}=\frac{\omega}{\sqrt{F}} x, \tilde{p}=\frac{\sqrt{F}}{\omega} p$, $\tilde{\xi}_{j}=\sqrt{\frac{\omega}{F}} \xi_{j}$ and $\tilde{\pi}_{j}=\sqrt{\frac{F}{\omega}} \pi_{j}$. Writing the equation (21) in terms of these dimensionless quantities and dropping the "tildes" to simplify the notation, we get

$$
\begin{aligned}
P_{j}^{\text {sink }} & \left(\xi_{j f}, T \mid \xi_{j 0}, 0 ; p\right) \\
= & \int_{\xi_{j}(0)=\xi_{j 0}}^{\xi_{j}(T)=\xi_{j f}} \mathcal{D} \xi_{j}(t) \int \mathcal{D} \pi_{j}(t) \\
& \times e^{-\int_{0}^{T} d t}\left\{p^{2} \xi_{j}^{2}(t)+\pi_{j}^{2}(t)+i \pi_{j}(t)\left(\dot{\xi}_{j}(t)+\xi_{j}(t)\right)\right\}
\end{aligned}
$$

As the action of the path integral in above equation is quadratic, it can be easily evaluated. Interestingly, $P_{j}^{s i n k}\left(\xi_{j f}, T \mid \xi_{j 0} ; p\right)$ is the probability of finding the $j^{t h}$ Brownian oscillator at $\xi_{j f}$ in presence of a sink which absorbs it at the position $\xi_{j}$ at a rate equal to $p^{2} \xi_{j}^{2}$. Thus the problem of diffusing diffusivity has been reduced to the problem of Brownian motion with absorption in $n$-dimensional space, ${ }^{54,55}$ a problem for which exact solution is easy to find. Since $P_{j}^{\text {sink }}\left(\xi_{j f}, T \mid \xi_{j 0} ; p\right)$ represents the propagator for Brownian motion of a harmonic oscillator in the overdamped limit, in presence of a $\operatorname{sink}^{54,55}$ which absorbs it at a rate $p \xi_{j}^{2}$, it obeys the Fokker-Planck equation 


$$
\left(\frac{\partial}{\partial t}-\frac{\partial^{2}}{\partial \xi_{j}^{2}}-\frac{\partial}{\partial \xi_{j}} \xi_{j}+p^{2} \xi_{j}^{2}\right) P_{j}^{\operatorname{sink}}\left(\xi_{j}, t \mid \xi_{j 0} ; p\right)=0
$$

with the initial condition

$$
P_{j}^{s i n k}\left(\xi_{j}, 0 \mid \xi_{j 0} ; p\right)=\delta\left(\xi_{j}-\xi_{j 0}\right) .
$$

This can be solved to get the solution at the final time $t=T$ to get

$$
\begin{aligned}
& P_{j}^{\operatorname{sink}}\left(\xi_{j f}, T \mid \xi_{j 0} ; p\right) \\
&= \frac{\sqrt{\alpha}}{2 \sqrt{\pi} \sqrt{\sinh (\alpha T)}} e^{\frac{T}{2}-\frac{\xi_{j f}^{2}}{4}+\frac{\xi_{j 0}^{2}}{4}} \\
& \quad \times e^{-\frac{1}{4} \alpha \operatorname{csch}(\alpha T)\left\{\left(\xi_{j f}^{2}+\xi_{j 0}^{2}\right) \cosh (\alpha T)-2 \xi_{j f} \xi_{j 0}\right\}},
\end{aligned}
$$

where

$$
\alpha=\sqrt{1+4 p^{2}} .
$$

The initial equilibrium distribution of the Brownian oscillator is obtained by (see Eq.(18)) taking the limit $T \rightarrow \infty$, which leads to

$$
\pi_{j, e q}\left(\xi_{j 0}\right)=\sqrt{\frac{1}{2 \pi}} \exp \left(-\frac{\xi_{j 0}^{2}}{2}\right) .
$$

Substituting for $P_{j}^{\text {sink }}\left(\xi_{j f}, T \mid \xi_{j 0} ; p\right)$ and $\pi_{j, e q}\left(\xi_{j 0}\right)$ from these expressions into Eq.(20), we get

$$
P(x, T)=\int_{-\infty}^{\infty} \frac{d p}{2 \pi} e^{-i p x} \bar{P}(p, T),
$$

with

$$
\bar{P}(p, T)=\left(\frac{4 \alpha e^{-(\alpha-1) T}}{(\alpha+1)^{2}-(\alpha-1)^{2} e^{-2 \alpha T}}\right)^{n / 2} .
$$

In a recent paper by Chechkin et. al. ${ }^{48}$ this result was obtained using the method of subordination.

The MSD is

$$
\left\langle x^{2}(T)\right\rangle=-\left(\frac{\partial^{2} \bar{P}(p, T)}{\partial p^{2}}\right)_{p=0}=2 n T,
$$

which shows that diffusion is Fickian for any $T$ and for all values of $n$. We now consider only the case $n=2$, as this has resemblance with the model of Chubynsky and Slater. ${ }^{49}$ This resemblance is made explicit in the next section where we also discuss the general case with arbitrary value of $n$.

For the case of $n=2$, we get

$$
\bar{P}(p, T)=\frac{4 \alpha e^{-(\alpha-1) T}}{(\alpha+1)^{2}-(\alpha-1)^{2} e^{-2 \alpha T}} .
$$

Further, in small $T$ limit, i.e. $\alpha T<<1$, the tail behavior of the distribution $P(x, T)$ is governed by,

$$
\bar{P}(p, T) \sim \frac{1}{1+2 p^{2} T} .
$$

This leads to an exponential form of $P(x, T)$. On the other hand, if $T>>1$, then

$$
\bar{P}(p, T) \sim e^{-T(\alpha-1)},
$$

which behaves like $e^{-2 p^{2} T}$ for small $p$ values and like $e^{-2 T|p|}$ for large $p$ values. Therefore, $P(x, T)$ resembles a Gaussian distribution for large $x$ and it resembles a Cauchy distribution for small $x$.

Non-Gaussianity Parameter: The deviation of the distribution from Gaussian is usually quantified by non-Gaussianity parameter. The parameter is zero for Gaussian distribution and is non-zero if the distribution is non-Gaussian. This parameter is defined as

$$
\gamma(T)=\frac{1}{3} \frac{\left\langle x^{4}(T)\right\rangle}{\left(\left\langle x^{2}(T)\right\rangle\right)^{2}}-1 .
$$

From equations (29) and (26), we get

$$
\left\langle x^{4}(T)\right\rangle=\left.\frac{\partial^{4} \bar{P}}{\partial p^{4}}\right|_{p=0}=12 n\left(n T^{2}+2 T+e^{-2 T}-1\right)
$$

Thus, the non-Gaussianity parameter, $\gamma(T)$ becomes

$$
\gamma(T)=\frac{2}{n T}-\frac{1}{n T^{2}}\left(1-e^{-2 T}\right) .
$$

In the limit, $T \rightarrow 0$, we get

$$
\lim _{T \rightarrow 0} \gamma(T)=2 / n \text {. }
$$

This corresponds to an exponential distribution if $n=2$. On the other hand in the limit of large $T$, we get

$$
\lim _{T \rightarrow \infty} \gamma(T)=0,
$$

which corresponds to a Gaussian distribution for all values of $n=1,2,3, \cdots$.

Figure 1 shows the evolution of non-Gaussianity parameter with time for various values of $n$. From these plots, it may be noticed that with an increase in the value of $n$, the non-Gaussianity starts disappearing sooner. In

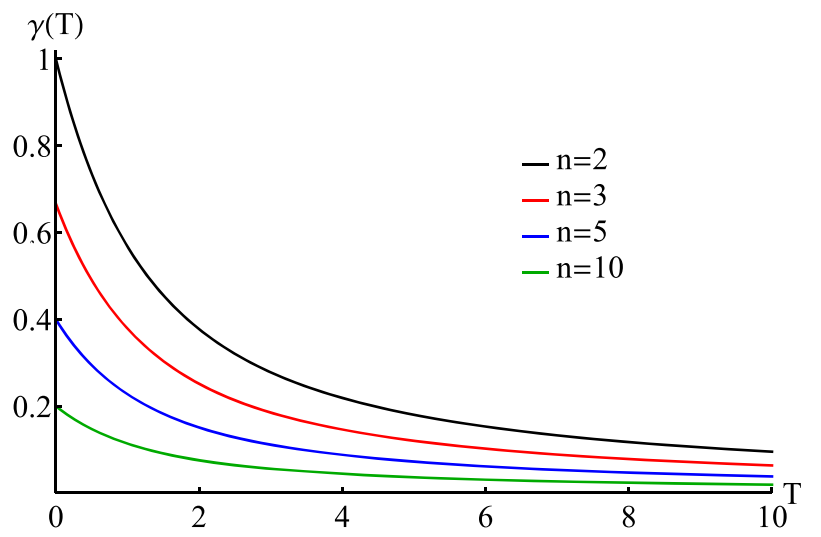

Figure 1. Non-Gaussianinty parameter as a function of $T$. 
other words, for higher $n$, the Gaussian behavior is established earlier.

\section{Relation to Chubynsky-Slater model}

Chubynsky and Slater, in their "diffusing diffusivity" model, ${ }^{49}$ assumed that in a complex system, viz diffusive motion of beads in lipid tubules ${ }^{27}$ etc., $D$ undergoes a biased random walk. Since, $D$ now changes randomly, the distribution of diffusivity, $\pi(D, t)$ follows an advection-diffusion equation,

$$
\frac{\partial \pi}{\partial t}=\frac{\partial}{\partial D}\left\{\frac{\partial}{\partial D} d(D)+s(D)\right\} \pi(D, t),
$$

where $d(D)$ is the diffusivity of the diffusion coefficient $D$ and $s(D)$ is the bias or the drift. They also argued that at short times such that $t<<\tau_{D}$ (where $\tau_{D}$ is the time scale for the diffusion of diffusivity or in other words it is the time scale for environment rearrangement), the diffusion of particle can be assumed constant. Thus on such small time scales, the pdf of particle displacements does not depend on the diffusion of diffusivity but only on its distribution. Now, for the simplest case, if one chooses a constant pair of $s$ and $d$, the resultant equilibrium distribution from Eq.(32) becomes exponential, $\pi_{e q}(D)=\frac{1}{D_{0}} e^{-D / D_{0}}$ with $D_{0}=s / d$. The argument given above thus leads to an exponential distribution of particle displacements at short times, i.e.,

$$
\begin{aligned}
P(x, T) & =\int_{0}^{\infty} d D \pi_{e q}(D) \frac{e^{-\frac{x^{2}}{4 D T}}}{\sqrt{4 \pi D T}} \\
& =\frac{1}{\sqrt{4 D_{0} T}} e^{-\frac{|x|}{\sqrt{D_{0} T}}} .
\end{aligned}
$$

Therefore, at short times Chubynsky and Slater were able to reproduce analytically the experimental observations made by Granick's group. ${ }^{27,28}$ But for intermediate to large times, they had to do simulations. However, with the present model of diffusing diffusivity, we have obtained exact results for all the time limits. In the following we consider the case of $n=2$, as this case leads to an equilibrium distribution for $D$ which is exponential and therefore has resemblance with the model of Chubynsky and Slater. The general case with arbitrary value of $n$ is also discussed.

\section{$4.1 \quad n=2$ case}

Assuming $D(t)=\xi_{1}^{2}+\xi_{2}^{2}$, where $\left\{\xi_{1}, \xi_{2}\right\}$ are the position coordinates of a two dimensional isotropic, Brownian harmonic oscillator, we can write a Fokker-Planck (FP) equation for $D .{ }^{56}$ The FP equation for the distribution of $D$ in this case is

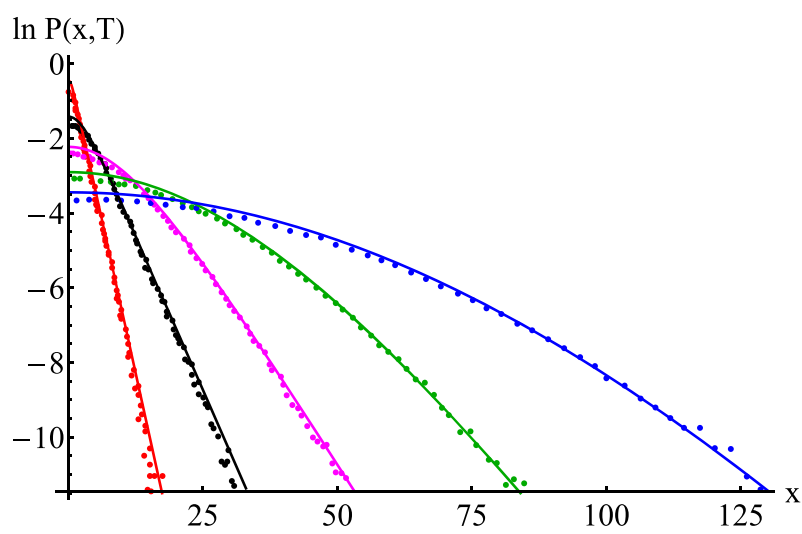

Figure 2. Comparison of the pdf at different times with the simulation data of Chubysnky and Slater. ${ }^{49}$ The dots represent the data extracted from Figure 1 of ref. [28] and solid lines are the plots obtained using the analytical expressions in equations (28) and (29).

$$
\begin{aligned}
\frac{\partial}{\partial t} \pi(D, t)= & \frac{\partial}{\partial D}\left\{4 F \frac{\partial}{\partial D} D+2 \omega\left(D-\frac{2 F}{\omega}\right)\right\} \\
& \times \pi(D, t) .
\end{aligned}
$$

This equation, although not identical with Eq.(32), gives an exponential steady-state distribution after assuming reflecting boundary condition at $D=0$, i.e.,

$$
\pi_{e q}(D)=\frac{1}{D_{0}} e^{-D / D_{0}}
$$

with $D_{0}=2 F / \omega$. This is same as in the model of Chubynsky and Slater. In the simulations of the diffusing diffusivity model, Chubynsky and Slater chose the values of the parameters as $d=0.0025$ and $s=0.01$ so that $D_{0}=0.25$. The simulation results of their model are presented by dots in the plots of Figure 2. In order to make a fair comparison with their model, we choose $F=0.0025$ and $\omega=0.02$ such that $D_{0}=0.25$. With these values of parameters, the plots for $P(x, T)$ are shown by solid lines in Figure 2. We see that there is very good agreement between the two models despite the fact that the underlying equations governing the evolution of diffusivity in two models are very different, see equations (32) and (33).

\subsection{The general case}

For the general case when $D(t)=\xi_{1}^{2}+\xi_{2}^{2}+\cdots+\xi_{n}^{2}$, it may be noticed that the equilibrium distribution for diffusivity is

$$
\pi_{e q}(D)=\frac{1}{D_{0}} \frac{1}{\Gamma(n / 2)}\left(\frac{D}{D_{0}}\right)^{\frac{n}{2}-1} e^{-D / D_{0}},
$$


which is a generalized gamma function. This result is not surprising as the sum of iid normal random variables, known as chi-squared distribution, ought to have a gamma distribution. Following a Slater-type argument, at short times, the pdf for displacements of particle is given as

$$
\begin{aligned}
P(x, T)= & \frac{1}{\Gamma(n / 2)} \frac{1}{\sqrt{\pi D_{0} T}}\left(\frac{x^{2}}{4 D_{0} T}\right)^{\frac{n-1}{4}} \\
& \times K_{-\frac{n}{2}+\frac{1}{2}}\left(\frac{|x|}{\sqrt{D_{0} T}}\right)
\end{aligned}
$$

where $K_{v}(z)$ is the modified Bessel function of second kind, of order $v .^{57}$

\section{Conclusions}

The problem of diffusing diffusivity has been shown to be equivalent to Brownian motion with absorption which leads to analytical solutions for the problem. The mean square displacement is shown to increase linearly with time at all the times, i.e., $\left\langle x(T)^{2}\right\rangle \propto T$. At short times, the distribution of displacements is found to be non-Gaussian while at long times, it crosses over to being Gaussian. We have also demonstrated that our analytical results reproduce the numerical results of Chubynsky and Slater ${ }^{49}$ quite well.

The model of "diffusing diffusivity" presented here is quite general and one can easily find exact analytical solution in the case where $D$ is modelled as the square of the distance of an n-dimensional harmonic oscillator from the origin. We further believe that the results obtained in this paper are not very sensitive to the choice of the model. Simple scaling arguement presented in a very recent paper ${ }^{12}$ shows that any model of "diffusing diffusivity" for translational diffusion would lead to Fickian behaviour as well as Gaussian distribution in the long time limit if the distribution for $D$ has finite first and second moments. However, if the first and second moments are not finite, different results can be expected. It was found in this paper ${ }^{12}$ that for a distribution of $D$ with a power-law of the form $D^{-1-\alpha}$, the pdf for displacements was a Lévy stable distribution with stability index $2 \alpha$. This leads to superdiffusion in the large $T$ limit. Further, given the amount of data available from experiments and also from computer simulations, it is a fair question to ask if these models of "diffusing diffusivity" can give rise to subdiffusive dynamics. This seems to be the case if one assumes a model where diffusivity obeys a timefractional Fokker Planck equation. In crowded media, we believe that it is quite likely that this would be the situation. We are currently investigating such interesting possibilities and our preliminary results show that the dynamics in this case is subdiffusive and nonGaussian.

\section{Acknowledgements}

We dedicate this paper to the memory of Prof. Charusita Chakravarty, who was a dear friend and a very capable theoretician. KLS is grateful to Professors Arindam Chowdhury and Rajarshi Chakrabarti for discussions. Both the authors thank J C Bose Fellowship of K L Sebastian for financial support.

\section{References}

1. Einstein A 1905 On the Movement of Small Particles Suspended in Stationary Liquids Required by the Molecular Kinetic Theory of Heat Ann. Phys. 17549

2. Chandrasekhar S 1943 Stochastic Problems in Physics and Astronomy Rev. Mod. Phys. 151

3. Frey E and Kroy K 2005 Brownian Motion: A Paradigm of Soft Matter and Biological Physics Ann. Phys. 1420

4. Sokolov I M 2012 Models of Anomalous Diffusion in Crowded Environments Soft Matter 89043

5. Höfling F and Franosch T 2013 Anomalous Transport in the Crowded World of Biological Cells Rep. Prog. Phys. 76046602

6. Metzler R, Jeon J-H, Cherstvy A G and Barkai E 2014 Anomalous Diffusion Models and Their Properties: Non-Stationarity, Non-Ergodicity, and Ageing at the Centenary of Single Particle Tracking Phys. Chem. Chem. Phys. 1624128

7. Ott A, Bouchaud J-P, Langevin D and Urbach W 1991 Anomalous diffusion in elongated micelles and its Levy flight interpretation J. Phys. II. (France) 11465

8. Bouchaud J-P and Georges A 1990 Anomalous diffusion in disordered media: Statistical mechanisms, models and physical applications Phys. Rep. 195127

9. Stauffer D, Schulze C and Heermann D W 2007 Superdiffusion in a model for diffusion in a molecularly crowded environment J. Biol. Phys. 33305

10. Ratynskaia S, Rypdal K, Knapek C, Khrapak S, Milovanov A V, Ivlev A, Rasmussen J J and Morfill G E 2006 Superdiffusion and viscoelastic vortex flows in a twodimensional complex plasma Phys. Rev. Lett. 96105010

11. Caspi A, Granek R and Elbaum M 2002 Diffusion and directed motion in cellular transport Phys. Rev. E 66 011916

12. Jain R and Sebastian K L 2017 Lévy flight with absorption: A model for diffusing diffusivity with long tails Phys. Rev. E 95032135

13. Golding I and Cox E C 2006 Physical nature of bacterial cytoplasm Phys. Rev. Lett. 96098102

14. Guigas G, Kalla C and Weiss M 2007 Probing the nanoscale viscoelasticity of intracellular fluids in living cells Biophys. J. 93316

15. Weber S C, Spakowitz A J and Theriot J A 2010 Bacterial chromosomal loci move subdiffusively through a viscoelastic cytoplasm Phys. Rev. Lett. 104238102 
16. Lampo T J, Stylianidou S, Backlund M K, Wiggins P A and Spakowitz A J 2017 Cytoplasmic RNA-protein particles exhibit non-gaussian subdiffusive behavior Biophys. J. 112532

17. Ernst D, Köhler J and Weiss M 2014 Probing the type of anomalous diffusion with single-particle tracking Phys. Chem. Chem. Phys. 167686

18. He W, Song H, Su Y, Geng L, Ackerson B J, Peng H $\mathrm{B}$ and Tong P 2016 Dynamic heterogeneity and nonGaussian statistics for acetylcholine receptors on live cell membrane Nat. Comm. 71

19. Polanowski P and Sikorski A 2014 Simulation of diffusion in a crowded environment Soft Matter 103597

20. Hasnain S, McClendon C L, Hsu M T, Jacobson M P and Bandyopadhyay P 2014 A new coarse-grained model for E. coli cytoplasm: accurate calculation of the diffusion coefficient of proteins and observation of anomalous diffusion PloS One 9 e 106466

21. Hasnain S and Bandyopadhyay P 2015 An analytical correlated random walk model and its application to understand subdiffusion in crowded environment $J$. Chem. Phys. 143114104

22. Metzler R and Klafter J 2000 The random walk's guide to anomalous diffusion: a fractional dynamics approach Phys. Rep. 3391

23. Klafter J and Sokolov I M 2011 First Steps in Random Walks: From Tools to Applications (New York: Oxford University Press)

24. Jeon J-H and Metzler R 2010 Fractional brownian motion and motion governed by the fractional Langevin equation in confined geometries Phys. Rev. E 81021103

25. Magdziarz M, Weron A, Burnecki K and Klafter J 2009 Fractional Brownian motion versus the continuous-time random walk: A simple test for subdiffusive dynamics Phys. Rev. Lett. 103180602

26. Meroz Y, Sokolov I M and Klafter J 2013 Test for determining a subdiffusive model in ergodic systems from single trajectories Phys. Rev. Lett. 110090601

27. Wang B, Anthony S M, Bae S C and Granick S 2009 Anomalous yet Brownian Proc. Natl. Acad. Sci. USA 10615160

28. Wang B, Kuo J, Bae S C and Granick S 2012 When Brownian Diffusion is not Gaussian Nat. Mater. 11481

29. Guan J, Wang B and Granick S 2014 Even HardSphere Colloidal Suspensions Display Fickian Yet NonGaussian Diffusion ACS Nano 83331

30. Banks D S, Tressler C, Peters R D, Höfling F and Fradin C. 2016 Characterizing Anomalous Diffusion in Crowded Polymer Solutions and Gels over Five Decades in Time with Variable-Lengthscale Fluorescence Correlation Spectroscopy Soft Matter 124190

31. Jeon H, Cho H W, Kim J and Sung B J 2014 NonGaussian Rotational Diffusion in Heterogeneous Media Phys. Rev. E 90042105

32. Kegel W K 2000 Direct Observation of Dynamical Heterogeneities in Colloidal Hard-Sphere Suspensions Science $\mathbf{2 8 7} 290$

33. Kwon G, Sung B J and Yethiraj A 2014 Dynamics in Crowded Environments: Is Non-Gaussian Brownian Diffusion Normal? J. Phys. Chem. B 1188128
34. Ghosh S K, Cherstvy A G and Metzler R 2015 Nonuniversal tracer diffusion in crowded media of non-inert obstacles Phys. Chem. Chem. Phys. 171847

35. Chakrabarti R, Kesselheim S, Košovan P and Holm C 2013 Tracer Diffusion in a Crowded Cylindrical Channel Phys. Rev. E 87062709

36. Samanta N and Chakrabarti R 2016 Tracer Diffusion in a Sea of Polymers with Binding Zones: Mobile vs. Frozen Traps Soft Matter 128554

37. Eaves J D and Reichman D R 2009 Spatial Dimension and The Dynamics of Supercooled Liquids Proc. Natl. Acad. Sci. USA 10615171

38. Weeks E R 2000 Three-Dimensional Direct Imaging of Structural Relaxation Near the Colloidal Glass Transition Science $\mathbf{2 8 7} 627$

39. Chaudhuri P, Berthier L and Kob W 2007 Universal Nature of Particle Displacements Close to Glass and Jamming Transitions Phys. Rev. Lett. 99060604

40. Bhattacharya S, Sharma D K, Saurabh S, De S, Sain A, Nandi A and Chowdhury A 2013 Plasticization of Poly(vinylpyrrolidone) Thin Films under Ambient Humidity: Insight from Single-Molecule Tracer Diffusion Dynamics J. Phys. Chem. B 1177771

41. Bhattacharya S, Sharma D K, De S, Mahato J and Chowdhury A 2016 Heterogeneity During Plasticization of Poly(vinylpyrrolidone): Insights From Reorientational Mobility of Single Fluorescent Probes J. Phys. Chem. B 12012404

42. Kim J, Kim C and Sung B J 2013 Simulation Study of Seemingly Fickian but Heterogeneous Dynamics of Two Dimensional Colloids Phys. Rev. Lett. 110047801

43. Saltzman E J and Schweizer K S 2008 Large-Amplitude Jumps and Non-Gaussian Dynamics in Highly Concentrated Hard Sphere Fluids Phys. Rev. E 77051504

44. Majumder S R, Diermeier D and Rietz T A 2009 Price Dynamics in Political Prediction Markets Proc. Natl. Acad. Sci. USA 106679

45. Silva A C, Prange R E and Yakovenko V M 2004 Exponential Distribution of Financial Returns at Mesoscopic Time Lags: A New Stylized Fact Physica A 344 227

46. Metzler R 2017 Gaussianity Fair: The Riddle of Anomalous yet Non-Gaussian Diffusion Biophys. J. 112413

47. Cherstvy A G and Metzler R 2016 Anomalous Diffusion in Time-Fluctuating Non-Stationary Diffusivity Landscapes Phys. Chem. Chem. Phys. 1823840

48. Chechkin A V, Seno F, Metzler R and Sokolov I M 2017 Brownian yet Non-Gaussian Diffusion: From Superstatistics to Subordination of Diffusing Diffusivities Phys. Rev. X 7021002

49. Chubynsky M V and Slater G W 2014 Diffusing Diffusivity: A Model for Anomalous, yet Brownian, Diffusion Phys. Rev. Lett. 113098302

50. Jain R and Sebastian K L 2016 Diffusion in a Crowded, Rearranging Environment J. Phys. Chem. B 120 3988

51. Jain R and Sebastian K L 2016 Diffusing Diffusivity: Survival in a Crowded, Rearranging and Bounded Domain J. Phys. Chem. B 1209215 
52. Janakiraman D and Sebastian K L 2012 Path-Integral Formulation for Lévy Flights: Evaluation of the Propagator for Free, Linear, and Harmonic Potentials in the Over- and Underdamped Limits Phys. Rev. E 86061105

53. Kamenev A 2011 In Field Theory of Nonequilibrium Systems (New York: Cambridge University Press)

54. Bagchi B and Fleming G R 1990 Dynamics of Activationless Reactions in Solution J. Phys. Chem. 949
55. Sebastian K L 1992 Theory of Electronic Relaxation in Solution: Exact Solution for a $\delta$-function Sink in a Parabolic Potential Phys. Rev. A 46 R1732

56. Risken H 1989 In The Fokker-Planck Equation $2^{\text {nd }}$ ed. (Berlin: Springer-Verlag)

57. Abramowitz M and Stegun I A 1972 In Handbook of Mathematical Functions (New York: Dover Publications) 